\title{
Benefícios da implementação de Práticas Integrativas e Complementares na qualidade de vida de idosos institucionalizados
}

\author{
Benefits of implementing Integrative and Complementary Practices in the quality of life of \\ institutionalized elderly people
}

Beneficios de implementar Prácticas Integrativas y Complementarias en la calidad de vida de los adultos mayores institucionalizados

Luana Batista Faria1*, Júlia de Souza Silva Monteiro', Maria Luiza Feitoza Nolasco Alves¹, Kemile Albuquerque Leão'.

\section{RESUMO}

Objetivo: Analisar as possíveis repercussões da deficiência cognitiva e do transtorno depressivo em ldosos Institucionalizados em Instituições de Longa Permanência para Idosos (ILPIs), e levantar estratégias não farmacológicas utilizadas para melhora da cognição e da qualidade de vida. Métodos: A pesquisa foi realizada nas bases eletrônicas da biblioteca virtual de saúde (BVS), nas quais foram selecionados artigos originais publicados nos últimos 5 anos. Os critérios de inclusão foram os artigos originais, em português e inglês e alinhados com o tema da investigação. Foram considerados como critérios de exclusão os artigos duplicados e de revisão ou aqueles que não se enquadravam ao tema proposto. Foram excluídos os trabalhos que não apresentavam ferramentas para avaliação dos transtornos de depressão ou déficit cognitivo ou sugestões de tratamento não farmacológico. Resultados: $O$ envelhecimento populacional é um problema de Saúde Pública devido à natureza multifatorial e suas consequências epidemiológicas, sociais e econômicas. Idosos residentes em asilos são, em sua grande maioria, frágeis e apresentam alta incidência de transtornos depressivos, sendo necessário implementar medidas de assistência social e de saúde. Considerações finais: Portanto, torna-se necessário o fortalecimento de ações multiprofissionais com o objetivo de minimizar os danos e contribuir para o bem-estar e saúde desses idosos.

Palavras-chave: Disfunção cognitiva, Transtorno depressivo, Saúde do idoso institucionalizado.

\begin{abstract}
Objective: To analyze the possible repercussions of cognitive impairment and depressive disorder in Institutionalized Elderly in Long Stay Institutions for the Elderly (ILPIs), and to identify non-pharmacological strategies used to improve cognition and quality of life. Methods: The research was carried out in the electronic databases of the virtual health library ( $\mathrm{VHL}$ ), in which original articles published in the last 5 years were selected. Inclusion criteria were original articles, in Portuguese and English and aligned with the research topic. Duplicate and review articles or those that did not fit the proposed theme were considered as exclusion criteria. Studies that did not present tools for the assessment of depression or cognitive deficit disorders or suggestions for non-pharmacological treatment were excluded. Results: Population aging is a Public Health problem due to its multifactorial nature and its epidemiological, social and economic consequences. Elderly residents in nursing homes are, for the most part, fragile and have a high incidence of depressive disorders, and it is necessary to implement social and health care measures. Final considerations: Therefore, it is necessary to strengthen multidisciplinary actions in order to minimize damage and contribute to the well-being and health of these elderly people.
\end{abstract}

Key words: Cognitive dysfunction, Depressive disorder, Health of institutionalized elderly.

\footnotetext{
${ }^{1}$ Faculdade Dinâmica do Vale do Piranga (FADIP), Ponte Nova - MG. *E-mail: farialu47@gmail.com
} SUBMETIDO EM: 2/2022 


\begin{abstract}
RESUMEN
Objetivo: Analizar las posibles repercusiones del deterioro cognitivo y el trastorno depresivo en Ancianos Instituidos en Instituciones de Larga Estancia para Ancianos (ILPI), e identificar estrategias no farmacológicas utilizadas para mejorar la cognición y la calidad de vida. Métodos: La investigación fue realizada en las bases de datos electrónicas de la biblioteca virtual en salud (BVS), en las cuales fueron seleccionados artículos originales publicados en los últimos 5 años. Los criterios de inclusión fueron artículos originales, en portugués e inglés y alineados con el tema de investigación. Se consideraron como criterios de exclusión artículos duplicados, de revisión o que no se ajustaran a la temática propuesta. Se excluyeron los estudios que no presentaron herramientas para la evaluación de la depresión o los trastornos por déficit cognitivo o sugerencias para el tratamiento no farmacológico. Resultados: El envejecimiento poblacional es un problema de Salud Pública por su carácter multifactorial y sus consecuencias epidemiológicas, sociales y económicas. Los ancianos residentes en residencias de ancianos son, en su mayoría, frágiles y con una alta incidencia de trastornos depresivos, siendo necesaria la implementación de medidas socio-sanitarias. Consideraciones finales: Por lo tanto, es necesario fortalecer las acciones multidisciplinarias para minimizar los daños y contribuir al bienestar y la salud de estos ancianos.
\end{abstract}

Palabras clave: Disfunción cogniva, Trastorno depresivo, Salud del anciano institucionalizado.

\title{
INTRODUÇÃO
}

O envelhecimento é uma etapa natural do ciclo de vida humano. No Brasil, o número de idosos écrescente: segundo o Instituto Brasileiro De Geografia e Estatística (IBGE) constatou-se 37,7 milhões de brasileiros acima dos 60 anos em 2021 e a tendência é que esse número aumente com o passar dos anos. Em quase todo o mundo a ampliação no número de idosos tem sido cada vez mais evidente, devido à elevação exponencial da expectativa de vida. É fato que o ser humano considera a longevidade importante; entretanto, é necessário que haja qualidade de vida (SANTOS PA, et al., 2019).

O idoso passa por um processo de envelhecimento cognitivo natural, conhecido como senescência, que envolve perda de funções psicológicas, fisiológicas e/ou anatômicas (SANTOS PA, et al., 2019). Como consequência do envelhecimento cognitivo, o aumento da sobrevida pode vir acompanhado de diversas outras situações, tais como o aumento da vulnerabilidade fisiológica relacionada à idade, alterações fisiológicas multissistêmicas, surgimento de doenças crônicas, diminuição de recursos sociais e financeiros concomitante com o aumento dos gastos com medicamentos e serviços de saúde e por fim, aumento de déficit cognitivo acompanhado por doenças mentais ou depressão, os quais constituem motivo de atenção pelos serviços de saúde (SOCIEDADE BRASILEIRA DE GERIATRIA E GERONTOLOGIA, 2021).

Segundo o Ministério da Saúde, a depressão pode ser classificada como uma doença e como um transtorno mental de múltiplas causas; independente da forma de classificação, o índice de depressão na população idosa é crescente, com maior prevalência em mulheres, pacientes com outras comorbidades ou pessoas institucionalizadas (SOUSA KA, et al., 2017).

As Instituições de Longa Permanência para Idosos (ILPIs) constituem um grande desafio: Apesar do lado positivo, já que muitas vezes são a única opção para idosos que não possuem família ou cuidadores e nem mesmo um lar fixo, para o idoso enquanto indivíduo, significa uma total mudança da sua rotina de vida. Além da saída do lar (ou do espaço habitual), do ambiente familiar, o idoso sof re com o af astamento das pessoas queridas e também enfrentará perda de liberdade, submetendo-se às regras da instituição (LIMA KVD, et al., 2017).

Diante desse contexto, o presente trabalho buscou levantar estudos sobre o diagnóstico de Deficiência Cognitiva e Transtorno Depressivo em idosos Institucionalizados em ILPIs e estabelecer discussões sobre depressão, Mini Exame do Estado Mental (MEEM), medicamentos e uso das práticas integrativas e complementares, contribuindo para a atualização acerca do tema. A proposta foi destacar a importância da assistência multidisciplinar aos idosos frente a um constante crescimento do envelhecimento populacional, somados à necessidade de evidenciar as vulnerabilidades da população senil. 


\section{MÉTODOS}

Trata-se de uma revisão bibliográfica realizada por meio de busca ativa na literatura referente à Deficiência Cognitiva e Transtorno Depressivo em idosos Institucionalizados em ILPI's. A busca de artigos foi realizada entre os meses de setembro e novembro do ano de 2021. A base de dados utilizada foi a biblioteca virtual de saúde (BVS).

Os critérios de inclusão foram: artigos originais, publicados nos últimos 5 anos, em língua portuguesa ou inglesa e alinhados com o tema da investigação. Foram excluídos os trabalhos que não apresentavam ferramentas para avaliação dos transtornos de depressão ou déficit cognitivo ou sugestões de tratamento não farmacológico.

Os descritores utilizados foram: depressão, idoso e Instituição de Longa Permanência para Idosos. O operador booleano utilizado foi AND. A partir desses descritores foram obtidos 788 artigos. A seguir, foram aplicados os critérios de inclusão, obtendo-se 380 artigos com texto completo, 358 nos idiomas português e inglês e por fim 139 artigos publicados nos últimos 5 anos. Foi realizada a leitura dos títulos e resumos destes 139 artigos. Dessa forma, após a leitura dos artigos na íntegra e da exclusão dos divergentes ou que não abordavam o tema proposto, 15 foram selecionados para a utilização da revisão, uma vez que atendiam aos critérios de inclusão.

Figura 1 - Fluxograma metodológico para seleção de artigos.

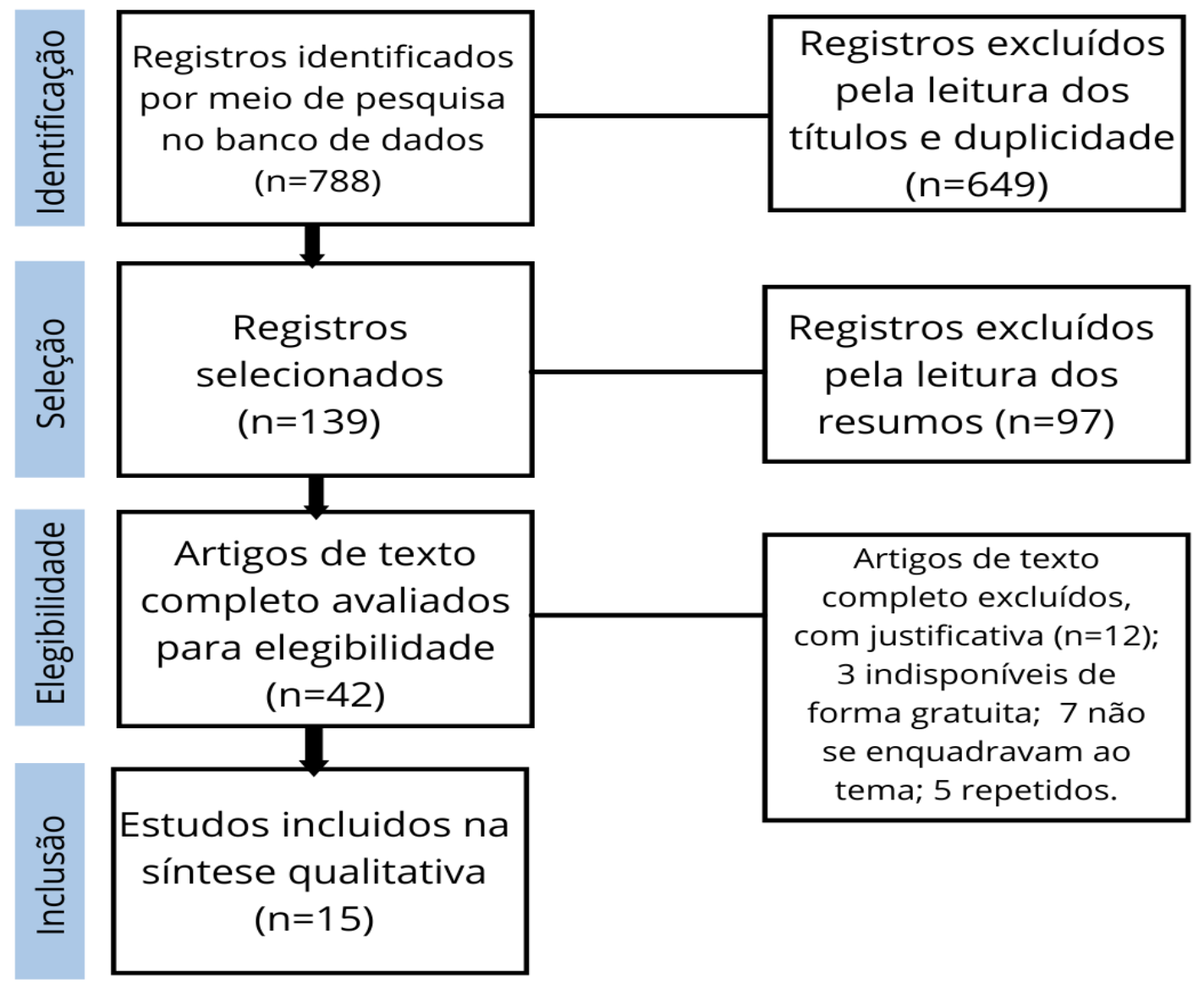

Fonte: FARIA LB, et al., 2022.

\section{RESULTADOS E DISCUSSÃO}

O Quadro 1 apresenta uma síntese dos artigos selecionados e estudados, com as principais informações extraídas dos mesmos. 
Quadro 1 - Análise de dados dos trabalhos selecionados.

\begin{tabular}{|c|c|c|c|c|}
\hline Autor & $\begin{array}{l}\text { Tipo de } \\
\text { estudo }\end{array}$ & $\begin{array}{c}\text { Local de } \\
\text { estudo }\end{array}$ & Objetivo & Resultados \\
\hline $\begin{array}{l}\text { MEDEIROS PA, } \\
\text { et al., } 2017 .\end{array}$ & $\begin{array}{l}\text { Revisão } \\
\text { Sistemática de } \\
\text { estudo } \\
\text { quantitativo }\end{array}$ & BRASIL & $\begin{array}{l}\text { Analisar de maneira ampla as evidências } \\
\text { científicas sobre a qualidade de vida dos } \\
\text { idosos residentes em instituições de longa } \\
\text { permanência, o que possibilitou a inclusão } \\
\text { de vários tipos de estudos observacionais, } \\
\text { com diferentes tipos de análises. }\end{array}$ & $\begin{array}{l}\text { Considera-se que um perfil de comorbidades, } \\
\text { incapacidade funcional, depressão e baixo suporte social, } \\
\text { somado às características organizacionais das instituições, } \\
\text { são as variáveis que mais influenciam na qualidade de vida } \\
\text { dos idosos residentes em instituições de longa permanência. }\end{array}$ \\
\hline $\begin{array}{l}\text { NOBREGA IP, } \\
\text { et al., } 2016 .\end{array}$ & $\begin{array}{l}\text { Estudo } \\
\text { Transversal }\end{array}$ & BRASIL & $\begin{array}{c}\text { Investigar a prevalência de sintomas } \\
\text { depressivos e fatores associados em idosos } \\
\text { institucionalizados no município de Recife, } \\
\text { Pernambuco. }\end{array}$ & $\begin{array}{c}\text { A alta prevalência de sintomas depressivos na população } \\
\text { institucionalizada alerta para a necessidade de maior } \\
\text { engajamento dos gestores e profissionais da saúde não só } \\
\text { na prevenção, como na investigação e valorização dos sinais } \\
\text { indicativos de depressão, a fim de que esta possa ser } \\
\text { precocemente diagnosticada e tratada da maneira mais } \\
\text { eficaz para o idoso. }\end{array}$ \\
\hline $\begin{array}{l}\text { VERÇOSA VSL, } \\
\text { et al., } 2016 .\end{array}$ & $\begin{array}{l}\text { Estudo } \\
\text { Transversal }\end{array}$ & BRASIL & $\begin{array}{l}\text { Identificar a presença de sintomatologia } \\
\text { depressiva em idosos que vivem em } \\
\text { instituições de longa permanência. }\end{array}$ & $\begin{array}{c}\text { A prevalência de sintomas depressivos foi de } 58 \% \text {; a maioria } \\
\text { mulheres }(53,8 \%) \text { e sem escolaridade }(69,2 \%) ; 75 \% \text { dos } \\
\text { idosos ref eriram presença de doença, as principais foram } \\
\text { hipertensão }(37,9 \%) \text { e diabetes mellitus }(27,3 \%) \text {. }\end{array}$ \\
\hline $\begin{array}{l}\text { ANDRADE C, et } \\
\text { al., } 2021 .\end{array}$ & $\begin{array}{l}\text { Estudo } \\
\text { qualitativo e } \\
\text { exploratório }\end{array}$ & BRASIL & $\begin{array}{l}\text { Rastrear indícios de depressão em idosos } \\
\text { residentes em instituições de longa } \\
\text { permanência (ILPI's). }\end{array}$ & $\begin{array}{l}\text { Detectou-se que idosos residentes da ILPI apresentaram } \\
\text { indícios para depressão, tendo como maior predisposição, os } \\
\text { idosos do sexo masculino (68\%) e os divorciados }(36 \%) \text {. }\end{array}$ \\
\hline $\begin{array}{l}\text { BRITO FM, et } \\
\quad \text { al, } 2021 .\end{array}$ & $\begin{array}{l}\text { Estudo } \\
\text { transversal }\end{array}$ & BRASIL & $\begin{array}{l}\text { Verificar a prevalência de sintomas } \\
\text { depressivos em pessoas idosas } \\
\text { institucionalizadas. }\end{array}$ & $\begin{array}{l}\text { Observou-se prevalência de depressão leve a moderada em } \\
57,1 \% \text { dos idosos institucionalizados. }\end{array}$ \\
\hline $\begin{array}{l}\text { CARVALHO PF, } \\
\quad \text { et al., } 2020 .\end{array}$ & $\begin{array}{l}\text { Estudo de } \\
\text { corte } \\
\text { transversal }\end{array}$ & BRASIL & $\begin{array}{l}\text { Identificar a presença de sintomas } \\
\text { depressivos e sua associação com a } \\
\text { autopercepção de saúde em idosos } \\
\text { residentes em instituições de longa } \\
\text { permanência. }\end{array}$ & $\begin{array}{c}\text { Dentre os idosos que apresentaram sintomas depressivos, } \\
32,3 \% \text { foram caracterizados como leves e } 13,4 \% \text { com } \\
\text { sintomas graves. Em relação à autopercepção de saúde, } \\
46,5 \% \text { consideraram sua saúde ruim/muito ruim. }\end{array}$ \\
\hline
\end{tabular}




\begin{tabular}{|c|c|c|c|c|}
\hline Autor & $\begin{array}{l}\text { Tipo de } \\
\text { estudo }\end{array}$ & $\begin{array}{l}\text { Local de } \\
\text { estudo }\end{array}$ & Objetivo & Resultados \\
\hline $\begin{array}{l}\text { SANTOS JO, et } \\
\text { al., } 2020 .\end{array}$ & $\begin{array}{l}\text { Estudo } \\
\text { exploratório } \\
\text { descritivo }\end{array}$ & BRASIL & $\begin{array}{l}\text { Mensurar a autoestima e o risco para } \\
\text { depressão em idosos residentes em } \\
\text { Instituição de Longa Permanência. }\end{array}$ & $\begin{array}{c}\text { Prevaleceram idosos com idade superior a } 70 \text { anos, sexo } \\
\text { feminino, viúvos, que possuíam filhos e visitados } \\
\text { mensalmente por familiares. Constatou-se que } 52 \% \text { estavam } \\
\text { com autoestima baixa e } 48 \% \text { estavam em risco para } \\
\text { depressão. }\end{array}$ \\
\hline $\begin{array}{c}\text { GUIMARÃES } \\
\text { LA, et al., } 2019 .\end{array}$ & $\begin{array}{l}\text { Estudo } \\
\text { epidemiológico } \\
\text { transversal }\end{array}$ & BRASIL & $\begin{array}{c}\text { Verificar a prevalência e fatores associados a } \\
\text { sintomas depressivos em idosos } \\
\text { institucionalizados. }\end{array}$ & $\begin{array}{c}\text { O estudo verificou alta prevalência de sintomas depressivos } \\
\text { em idosos institucionalizados, associado às variáveis } \\
\text { presença de incontinência urinária, autopercepção de saúde, } \\
\text { qualidade de sono e aposentadoria. }\end{array}$ \\
\hline $\begin{array}{l}\text { SCHERRER J, } \\
\text { et al., } 2019 .\end{array}$ & $\begin{array}{l}\text { Estudo } \\
\text { transversal }\end{array}$ & BRASIL & $\begin{array}{l}\text { Comparar a qualidade de vida dos idosos } \\
\text { residentes em lares para idosos com ou sem } \\
\text { sintomas de depressão. }\end{array}$ & $\begin{array}{c}\text { Sintomas de depressão alteraram negativamente a } \\
\text { qualidade de vida nos domínios: autonomia; atividades } \\
\text { presentes, passadas e futuras; participação social; } \\
\text { intimidade e pontuação total. }\end{array}$ \\
\hline $\begin{array}{c}\mathrm{ZHAO}, \mathrm{X} \text {, et al., } \\
2018 .\end{array}$ & $\begin{array}{l}\text { Estudo } \\
\text { transversal }\end{array}$ & CHINA & $\begin{array}{c}\text { Avaliar a prevalência de sintomas } \\
\text { depressivos, explorar se a resiliência mediou } \\
\text { a associação entre solidão e sintomas } \\
\text { depressivos e investigar se o suporte social } \\
\text { moderou o ef eito indireto ou direto do modelo } \\
\text { de mediação. }\end{array}$ & $\begin{array}{l}\text { A associação entre solidão e sintomas depressivos foi } \\
\text { parcialmente mediada pela resiliência. Além disso, o ef eito } \\
\text { indireto do modelo de mediação foi moderado pelo apoio } \\
\text { social. Os resultados sugerem que intervenções, como } \\
\text { melhorar a resiliência e o apoio social, podem ajudar a } \\
\text { quebrar a ligação entre a solidão e os sintomas. }\end{array}$ \\
\hline $\begin{array}{l}\text { GOMES JB, et } \\
\text { al., } 2016 .\end{array}$ & $\begin{array}{c}\text { Estudo } \\
\text { Transversal }\end{array}$ & BRASIL & $\begin{array}{c}\text { Avaliar os níveis de ansiedade e de } \\
\text { depressão em uma população de idosos } \\
\text { institucionalizados em municípios do interior } \\
\text { do estado da Bahia, Brasil. }\end{array}$ & $\begin{array}{l}\text { Os resultados indicaram idosos com algum tipo de dor, } \\
\text { sintomas de depressão leve e moderada, e frequentes } \\
\text { sintomas de ansiedade. }\end{array}$ \\
\hline $\begin{array}{l}\text { COELHO E, et } \\
\text { al., } 2020\end{array}$ & $\begin{array}{c}\text { Estudo } \\
\text { Longitudinal }\end{array}$ & PORTUGAL & $\begin{array}{c}\text { O objetivo deste estudo foi identificar os } \\
\text { ef eitos do movimento com a música sobre a } \\
\text { cognição e depressão em idosos } \\
\text { institucionalizados. }\end{array}$ & $\begin{array}{c}\text { Os resultados deste estudo mostraram melhoras } \\
\text { significativas na capacidade cognitiva e na função, } \\
\text { capacidade mental e depressão. Esses resultados estão em } \\
\text { congruência com a literatura existente, que afirma que os } \\
\text { programas de intervenção (seja treinamento cognitivo ou } \\
\text { atividade física, ou uma combinação de ambos) são eficazes } \\
\text { à manutenção e/ou melhoria do desempenho de habilidades } \\
\text { cognitivas e na redução da depressão. }\end{array}$ \\
\hline
\end{tabular}




\begin{tabular}{|c|c|c|c|c|}
\hline Autor & $\begin{array}{l}\text { Tipo de } \\
\text { estudo }\end{array}$ & $\begin{array}{l}\text { Local de } \\
\text { estudo }\end{array}$ & Objetivo & Resultados \\
\hline $\begin{array}{l}\text { HEIDARI M, et } \\
\text { al., } 2019\end{array}$ & $\begin{array}{l}\text { Estudo } \\
\text { Longitudinal }\end{array}$ & $\operatorname{IR} \tilde{A}$ & $\begin{array}{l}\text { O objetivo deste estudo foi investigar o ef eito } \\
\text { do cuidado espiritual sobre o estresse } \\
\text { percebido e a saúde mental dos idosos que } \\
\text { vivem no lar de idosos em lsfahan. }\end{array}$ & $\begin{array}{c}\text { Considerar a saúde espiritual como uma das dimensões da } \\
\text { saúde que influencia as variáveis psicossociais dos idosos } \\
\text { parece necessário. Os formuladores de políticas e } \\
\text { planejadores da área da saúde contribuem para a melhoria } \\
\text { do nível de saúde mental por meio da abordagem do cuidado } \\
\text { integral com ênfase na dimensão espiritual do cuidado ao } \\
\text { idoso. }\end{array}$ \\
\hline $\begin{array}{l}\text { CHING-TENG } \\
\text { Y, et al., } 2016\end{array}$ & $\begin{array}{l}\text { Estudo } \\
\text { Longitudinal }\end{array}$ & TAIWAN & $\begin{array}{l}\text { Este estudo testou a eficácia das atividades } \\
\text { de arteterapia na redução da depressão e na } \\
\text { melhora da autoestima de idosos residentes } \\
\text { em instituições de longa permanência. }\end{array}$ & $\begin{array}{c}\text { Os programas de arteterapia mostraram ef eitos promissores } \\
\text { na melhora da depressão e autoestima de idosos que vivem } \\
\text { em asilos. A incorporação de atividades artísticas no cuidado } \\
\text { do serviço social pode ajudar a desenvolver o cuidado de } \\
\text { longo prazo em uma direção mais diversificada, única e } \\
\text { inovadora. }\end{array}$ \\
\hline $\begin{array}{l}\text { ARRIETA H, et } \\
\text { al., } 2018\end{array}$ & $\begin{array}{l}\text { Estudo } \\
\text { experimental } \\
\text { randomizado }\end{array}$ & ESPANHA & $\begin{array}{l}\text { Examinar simultaneamente as mudanças no } \\
\text { desempenho físico, cognitivo e emocional ao } \\
\text { longo do processo de envelhecimento. }\end{array}$ & $\begin{array}{c}\text { A força muscular e a atividade física são fatores } \\
\text { positivamente associados a um melhor desempenho no } \\
\text { Teste de Aprendizagem Auditivo-Verbal de Rey, QV-AD e } \\
\text { Escala de Depressão de Goldberg em idosos com } \\
\text { comprometimento cognitivo leve a moderado residentes em } \\
\text { asilos. }\end{array}$ \\
\hline
\end{tabular}

Fonte: FARIA LB, et al., 2022. 
É sabido que o envelhecimento é um processo natural do organismo, que modifica a função fisiológica, cognitiva e até o desempenho de papéis sociais. Entretanto, o enfrentamento deste novo processo precisa ocorrer de forma eficaz e prazerosa para o idoso, já que o tratamento em si pode colocar em risco a sua saúde dos pacientes ao interferir na sua capacidade mental e funcional, além de interferir nas relações interpessoais e familiares (ANDRADE C, et al., 2021).

Em muitos casos, o idoso é encaminhado pela família para uma instituição de longa permanência devido à dificuldade financeira que enfrentam, o que limita a capacidade de oferecer o devido cuidado, na visão destes familiares. Entretanto, estas instituições nem sempre oferecem todos os serviços necessários para garantir a qualidade de vida dos idosos, acabando por contribuir com o isolamento, sedentarismo e restrição da vida social e af etiva dos pacientes idosos, visto que muitos deles recebem poucas ou até mesmo nenhuma visita. Este cenário favorece o surgimento de sintomas depressivos (MEDEIROS PA, et al., 2017).

Veras SMJ, et al. (2018) publicaram em seu estudo que o aumento da depressão nos asilados, pode ser desencadeado tanto pela conscientização de que o envelhecimento aproxima o indivíduo do final da vida como pela ausência de familiares, aumento da sensação de abandono e pelo surgimento de limitações físicas e incapacidades.

A depressão na terceira idade é comum, mas, ao contrário da opinião popular, não faz parte do processo natural do envelhecer. Ainda assim ela não pode ser facilmente detectada por ser classificada por muitos profissionais da saúde ou adultos empenhados no cuidado com idosos como um fenômeno natural da idade. É referido em diversos estudos que idosos moradores em instituições de longa permanência estão mais sujeitos a desenvolver depressão do que os idosos da comunidade, cercados por amigos e familiares. Existem evidências de que os idosos das casas de longa permanência vão continuar não sendo diagnosticados nas instituições que não possuam equipe profissional adequada para identificar esses fatores de risco (SANTOS JO, et al., 2020).

Verçosa VSL, et al. (2016) também apontaram que a depressão nos idosos institucionalizados pode ser desencadeada por diversos gatilhos, e envolve desde processos crônicos relacionados ao envelhecimento (doenças cardiovasculares, distúrbios endócrinos e autoimunes), polifarmácia (devido ao uso simultâneo de anti-hipertensivos, corticoides, medicamentos para neoplasias, dentre outros), até questões psicológicas ocasionadas pelo isolamento social, abandono, mudança no estilo de vida e déficit cognitivo.

Um dos fatores que estão diretamente ligados ao que se considera um bom envelhecimento é o ambiente familiar saudável, pois representa um porto seguro, importante na rotina do idoso. É no ambiente familiar que acontece a maior parte das interações, se fundamentam os vínculos e onde cada membro deve buscar exercer o seu papel, respeitando a individualidade do outro. Em grande parte dos trabalhos estudados foi possível perceber a associação entre os relatos de depressão e o descontentamento dos idosos em conviver com pessoas desconhecidas, perdendo subitamente parte de sua autonomia e contribuindo para o surgimento de um sentimento de abandono por seus familiares, fazendo com que estes idosos se sintam apenas mais um paciente dentro da instituição (ZHAO X, et al., 2018).

Estima-se que entre $14 \%$ e $22 \%$ dos idosos institucionalizados no mundo possuem depressão. No Brasil, essa estimativa chega a ser entre $21,1 \%$ e $61,6 \%$ (GUIMARÃES LA, et al., 2019). A importância de se investigar as origens da depressão em idosos institucionalizados se deve ao fato de o estado depressivo levar ao comprometimento funcional dos idosos asilados, resultando na perda da autonomia. O contexto institucional em que os idosos estão inseridos (longe dos familiares e sem af eto) pode contribuir de forma a aumentar a vulnerabilidade e posteriormente desencadear outras desordens psiquiátricas ou levar ao agravamento de patologias pré-existentes (BRITO FM, et al, 2021).

Com relação ao diagnóstico e tratamento da depressão nas instituições de longa permanência, foram observados vários relatos sobre a dificuldade inerente à esta tarefa. Ainda que se saiba da alta prevalência de depressão em idosos institucionalizados, a quantidade de fatores desencadeadores do transtorno depressivo torna o tratamento desafiador. A manif estação clínica mais relevante é a de início tardio, associado aos distúrbios cognitivos e ao pior desempenho nos testes neurológicos (SCHERRER GJ, et al., 2019). 
É importante destacar que a detecção precoce dos sintomas depressivos é importante para evitar o desenvolvimento do quadro depressivo, assim, prevenindo seus ef eitos negativos para a saúde e qualidade de vida desses idosos. No entanto, os profissionais de saúde que ali atuam devem estar aptos para uma prática dirigida não apenas para detectar sintomas de depressão, mas qualquer outra alteração que o idoso possa apresentar, entendendo que velhice não deve ser encarada como sinônimo de doença (VERÇOSA VSL, et al., 2016).

Vários estudos apontam uma relação entre o déficit cognitivo e a depressão em idosos (CARDOSO RL, et al., 2020; PAIXÃO YA, et al., 2019). Por esta razão, a avaliação da função cognitiva precisa ser incluída durante a investigação da depressão. O MEEM é um instrumento que tem como objetivo rastrear perdas cognitivas em quadros demenciais, utilizado no Brasil e em todo o mundo, sendo o mais empregado para avaliar as funções cognitivas (ANDRADE C, et al, 2021).

Este teste avalia a memória de curto prazo, a atenção, o cálculo e as habilidades de linguagem. Consiste em duas etapas, sendo a primeira etapa relacionada à avaliação da memória e a segunda etapa exigindo leitura, escrita e comandos verbais, totalizando um score de 30 pontos. Pacientes analfabetos possuem a nota de corte de 18 a 19 pontos; idosos que possuem entre 1 a 3 anos de escolaridade, 23 pontos; idosos com 4 a 7 anos de escolaridade, 24 pontos e idosos com mais de 7 anos de escolaridade, 28 pontos. É importante ressaltar que quando reaplicado o MEMM, caso os resultados apontem valores mais baixos que a pontuação anterior, isso indica que há um certo risco de déficit cognitivo (GOMES JB e REIS LA, 2016).

Embora o MEEM seja uma ferramenta útil no rastreio de doenças cognitivas, sua realização muitas vezes é de extrema dificuldade. Muitos dos idosos que residem nas ILPIs estão com a saúde debilitada, acamados, não dialogam muito e não apresentam uma boa orientação de tempo e espaço, isso faz com que as questões propostas no MEEM não possam ser respondidas ou quando são, não apresentam um resultado fidedigno para a escala (GAMA DET, et al., 2021).

Um outro aspecto que também pôde ser observado é o fato de o processo de envelhecimento trazer consigo uma série de doenças crônico-degenerativas que tornam necessário o uso de diversos medicamentos, sendo frequente o relato de polifarmácia em idosos, e ainda mais frequente em idosos institucionalizados (ROCHA VC, et al., 2019). Além disso, é comum nesta faixa etária o uso concomitante de antidepressivos e antipsicóticos. Os antidepressivos são medicamentos eficazes no tratamento da depressão em idosos, sendo os Antidepressivos Tricíclicos (ADT), como a Nortriptilina e Desipramina, considerados padrão-ouro para o tratamento (PRADO MAMP, et al., 2017).

Entretanto, os antidepressivos possuem diversos efeitos colaterais, incluindo prejuízo da memória, quadros de delirium e alterações cardiovasculares. Por estas razões, devem ser prescritos com cautela. Apesar da comprovada eficácia dos antidepressivos e dos antipsicóticos, deve-se sempre refletir sobre o risco-benefício na inserção de múltiplos medicamentos em pacientes com a saúde já debilitada, correndo-se o risco de sobrecarregar ainda mais o sistema hepático e o sistema renal. Assim sendo, torna-se importante a investigação de medidas não farmacológicas que possam ser utilizadas para a melhoria da qualidade de vida dos idosos e minimizar o uso de medicamentos, restringindo as prescrições a situações específicas (OLIVEIRA HSB e CORRADI MLG, 2018).

O mérito das Práticas Integrativas e Complementares está em compreender o ser humano como um ser integral, não identificando obstáculos entre mente, corpo e espírito, em contrapartida do que faz a medicina convencional). Por trabalhar a saúde dos pacientes visando um bem-estar amplo, de fatores físicos, sociais, mentais, emocionais e espirituais, objetivando uma melhoria na qualidade de vida dos pacientes, estas práticas podem ser extremamente benéficas para os idosos institucionalizados (TESSER DC, et al., 2018).

A Política Nacional de Práticas Integrativas e Complementares (PNPIC), foi estabelecida em 2006 no Sistema Único de Saúde (SUS), através da Portaria GM/MS no 9712. Essa portaria orienta a estrutura das Práticas Integrativas e Complementares nos serviços da Atenção Básica. A PNPIC surgiu decorrente das demandas sociais e institucionais e engloba 29 práticas diferentes. São elas: Medicina Tradicional Chinesa/Acupuntura, Medicina Antroposófica, Homeopatia, Plantas Medicinais e Fitoterapia, Termalismo Social/Crenoterapia, Arteterapia, Ayurveda, Biodança, Dança Circular, Meditação, Musicoterapia, 
Naturopatia, Osteopatia, Quiropraxia, Reflexoterapia, Reiki, Shantala, Terapia Comunitária Integrativa, Yoga, Apiterapia, Aromaterapia, Bioenergética, Constelação familiar, Cromoterapia, Geoterapia, Hipnoterapia, Imposição de mãos, Ozonioterapia e Terapia de Florais (MINISTÉRIO DA SAÚDE, 2006).

É importante frisar que existem algumas limitações. Primeiro, nem todas as práticas constantes na PNPIC visam tratar a depressão. Além disso, grande parte dos municípios brasileiros ainda não foi capaz de implementar nem mesmo algumas das Práticas Integrativas no Sistema de Saúde municipal. Por último, ainda há poucos trabalhos publicados expondo os benefícios das Práticas Integrativas em Instituições de Longa Permanência (JUNIOR ET, 2016).

Um estudo publicado revelou que o uso de música associada ao movimento levou idosos a apresentar uma melhora significativa na função cognitiva, na capacidade mental e no quadro de depressão. Esses resultados estão em congruência com a literatura existente, que afirma que os programas de intervenção (seja cognitivo-treinamento ou atividade física, ou uma combinação de ambos) são eficazes e levam à manutenção e / ou melhoria no desempenho das habilidades cognitivas, e redução dos níveis de depressão. A música mobiliza quase todas as funções cognitivas e o processamento musical envolve diferentes regiões, incluindo todos os lobos, estruturas corticais e subcorticais (COELHO E, et al., 2020).

Heidari M, et al. (2019), apontaram que a espiritualidade e as intervenções espirituais são eficazes na manutenção e melhoria da saúde dos idosos. Dada a experiência de vazio e isolamento espiritual entre os idosos, o cuidado espiritual é considerado como um elemento-chave para alcançar a integridade individual, melhorando a comunicação consigo mesmo, com o meio ambiente e a natureza. A criação artís tica é um outro exemplo de medida não farmacológica que fornece aos idosos um método de comunicação não verbal; promove oportunidades para auto-retrospecto, autorreflexão e interações interpessoais; e, além disso, permite que eles expressem seus sentimentos, compreendam a si mesmos, relaxem suas emoções e adquiriram novas habilidades, além de uma sensação de felicidade. Por meio desses benefícios, artes e ofícios aliviam ansiedade, depressão, hostilidade, sensação de solidão e isolamento social e aumentam a autoestima e a alegria de viver (CHING-TENG Y, et al., 2016).

Estes são apenas alguns relatos dos benefícios proporcionados aos idosos após a implementação de práticas integrativas e complementares em instituições de longa permanência. Sabe-se que práticas como a Aromaterapia, Terapia de Florais, dentre outras, possuem eficácia em quadros depressivos e trariam grandes benefícios aos idosos institucionalizados. Entretanto, é preciso vencer as principais barreiras para implementação destas práticas, a saber: profissionais capacitados, custos com material e regulamentação junto ao sistema de saúde municipal (WANNMACHER L, 2016).

Investir na qualificação dos profissionais que atuam no âmbito das ILPI é essencial, não apenas para a implementação de práticas integrativas e complementares, mas também para que estes profissionais estejam preparados para identificar sintomas indicadores de depressão entre os institucionalizados, atuando de maneira eficiente na prevenção, no controle e no tratamento dessa patologia. Sugere-se, portanto, que a investigação da depressão em idosos ocorra de forma individualizada, por meio de métodos de avaliação e de instrumentos específicos do campo da Gerontologia e que levem em consideração os contextos clínico, psíquico, familiar e social nos quais o idoso se encontra para uma tomada de decisão adequada com relação ao tratamento destes idosos (NÓBREGA IP, et al.,2016).

É essencial que as instituições de longa permanência possam contar com uma equipe multidisciplinar de saúde, formada por profissionais que atuem na saúde do idoso, sendo eles médicos, nutricionistas, enfermeiros, farmacêuticos, psicólogos e fisioterapeutas. Cada membro da equipe deve desempenhar um papel primordial e todos devem buscar interagir com os idosos e, através de seus conhecimentos, intervir a fim de of erecer-lhes uma melhor qualidade de vida, reduzindo os impactos psicológicos e sociais e of erecendo um ambiente seguro e acolhedor (GUIMARÃES LA, et al., 2019).

Deve-se sempre ter em mente o contexto da institucionalização dos idosos, atentando-se ao fato de os sinais e sintomas depressivos estarem relacionados à insatisfação dos idosos por estar lo nge de seus familiares e terem que conviver com o desconhecido, seguindo uma rotina de horários para suas atividades cotidianas imposta por terceiros, perdendo assim a autonomia e gerando um sentimento de inferioridade, 
como se ele fosse apenas mais um dentro da Instituição de longa permanência. Por isso, se torna indispensável a atuação de uma equipe multidisciplinar qualificada (GAMA DET, et al., 2019).

\section{CONSIDERAÇÕES FINAIS}

Com este estudo foipossível perceberque como aumento do envelhecimento populacional em nosso país nos últimos anos, torna-se necessário implementar medidas de prevenção para o declínio funcional e cognitivo dos idosos. Está claro que a depressão não é uma consequência natural do envelhecimento e que promove graves consequências, como o sofrimento psíquico, redução e perda dos relacionamentos interpessoais, prejuízo nas atividades de vida diária e aumento dos gastos em saúde. Evidenciou-se que a depressão interfere de forma significativa, não apenas no bem-estar fisiológico do indivíduo, mas também na vida social. Por meio de uma equipe multidisciplinar qualificada e disposta a implementar medidas não farmacológicas como as práticas integrativas e complementares, é possível abordar a saúde do idoso de forma integral, avaliando seus aspectos biológicos, psicológicos e sociais, a fim de preservar o grau de independência funcional.

\section{REFERÊNCIAS}

1. ANDRADE CS, et al. Rastreamento de depressão em idosos residentes em instituições de longa permanência. Nursing, 2021;24:6179-6190.

2. ARRIETA $\mathrm{H}$, et al. Physical activity and fitness are associated with verbal memory, quality of life and depression among nursing homem residents: preliminary data of randomized controlled trial. BMC Geriatr, 2018; 18: 80.

3. BRITO FM, et al. Diagnóstico de enfermagem regulação do humor prejudicada e sintomas depressivos em pessoas institucionalizadas. Pesquisa (Univ. Fed. Estado Rio J., online), 2021;13:919 -924.

4. CARDOSO RL, et al. Disfunção cognitiva da depressão: O triste esquecimento. Gazeta Médica, 2020; 7: 348 - 354.

5. CHING-TENG Y, et al. Positive effects of art therapy on depression and self-esteem of older in nursing homes. Social Work in Health Care, 2019; 58: 324-338.

6. COELHO E, et al. Exercise with music: An Innovative Approach to Increase Cognition and Reduce Depression in Institutionalized Elderly. Psicologia del Deporte, 2020;29: 49-56.

7. GAMA DET, et al. A funcionalidade de idosos institucionalizados:uma revisão integrativa. Acervo Saúde, 2021; 13 : 1-9.

8. GOMES JB, REIS LA. Descrição dos sintomas de ansiedade e depressão em idosos institucionalizados no interior da Bahia, Brasil. Kairós, 2016; 19: 175-191

9. GUIMARÃES LA, et al. Sintomas depressivos e fatores associados em idosos residentes em instituição de longa permanência. Ciência e Saúde Coletiva, 2019;24: 3276-3282.

10. HEIDARI M, et al. The Effect of spiritual care on perceived stress and mental health among the elderlies living in Nursing Home. J Reling Health, 2019; 58: 1328- 1339.

11. JUNIOR ET. Práticas Integrativas e Complementares em Saúde, uma nova eficácia do SUS. Estudos Avançados, 2016;30: 99 - 112.

12. LIMA KVD, et al. Instituição de longa permanência como alternativa no acolhimento das pessoas idosas. Ver. Salud Pública, 2017;19: $210-214$.

13. MEDEIROS PA, et al. Avaliação da qualidade de vida de idosos institucionalizados: revisão sistemática de estudos qualitativos. Pensar a prática, 2017;20: 150-171.

14. MINISTÉRIO DA SAÚDE. Portaria nำ71, 3 de Maio de 2006. Aprova a Política Nacional de Práticas Integrativas e Complementares (PNPIC) no Sistema Único de Saúde. Brasília, 2006. Disponível em: http://www.crbm 1.gov.br/Portaria\%20MS\%20971\%202006.pdf.Acessado em:9 setembro 2021.

15. NOBREGA IP, et al. Prevalência de sintomas depressivos e fatores associados em idosos institucionalizados no município de Recife, Pernambuco. Estudos interdisciplinares sobre o envelhecimento, 2016;21:135-154.

16. OLIVEIRA HSB, CORRADI MLG. Aspectos farmacológicos do idoso:uma revisão integrativa de literatura. Rev Med, 2018;97: 165-176.

17. OLIVEIRA L, GONÇALVES JR. Depressão em idosos institucionalizados:uma revisão de literatura. Revista JRG de estudos acadêmicos, 2020;3: 110-122.

18. PAIXÃO YA, et al. Declínio cognitivo e sintomas depressivos: Um estudo com idosos da Universidadeda Maturidade. 2019;6: $121-127$.

19. PRADO MAMP, et al. Uso de medicamentos psicotrópicos em adultos e idosos residentes em Campinas, São Paulo: um estudo transversal de base populacional. Epidemiol. Serv. Saúde, 2017;26: 1 -9.

20. ROCHA VC, et al. Prevalência de medicamentos neuropsiquiátricos utilizados por idosos institucionalizados em Boa Vista/RR. Congresso Internacional de Envelhecimento Humano, 2019;2: 1 - 6.

21. SANTOS JO, et al. Autoestima e risco para depressão em idosos residentes em instituições de longa permanência. Espaço para a saúde, 2020;21:59-70. 
22. SANTOS PA, et al. A percepção do idoso sobre a comunicação no processo de envelhecimento. Audiology Communication Research, 2019;24:1-8.

23. SCHERRER J, et al. Quality of life of institutionalized aged with and without symptoms of depression. Rev. Bras. Enferm, 2019;72: 127-133.

24. SOCIEDADE BRASILEIRA DE GERIATRIA E GERONTOLOGIA. Atenção integral à saúde da pessoa idosa:o papel da equipe multidisciplinar. 2021. Disponível em: https://sbgg.org.br/atencao-integral-a-saude-da-pessoa-idosa-opapel-da-equipe-multidisciplinar/. Acessado em: 10 setembro 2021.

25. SOUSA KA, et al. Prevalência de sintomas de depressão em idosos assistidos pela estratégia de saúde da família. Rev. Min. Enferm., 2017;21:1-6.

26. TESSER DC, et al. Práticas Integrativas e Complementares na Atenção primária à Saúde Brasileira. Saúde Debate, 2018;42: $174-188$.

27. VERAS SMJ, et al. Produção científica sobre saúde mental de idosos residentes em instituições de longa permanência. ID on line Revista de Psicologia, 2018;12:336-352.

28. VERÇOSA VSL, et al. Prevalência de sintomatologia depressiva em idosos institucionalizados. Revista de Enfermagem, 2016;10:4264-4270.

29. WANNMACHER L. Abordagem da depressão maior em idosos: medidas não medicamentosas e medicamentosas. Organização Pan-Americana da Saúde, 2016;1:1-10.

30. ZHAO X, et al. Loneliness and depression symptoms among the elderly in nursing homes: A moderated medi ation model of resilience and social support. Res Psychiatry, 2018;268:143-151. 